Editorial

\title{
Document related evidence at the scene of a crime
}

\section{Editorial}

Documents are often overlooked at a crime scene, usually by the overzealous investigator. Often, documents can give a history or help explain the basis for a crime. They can be in any form as people in the field know that a document is anything bearing the thought of "man." In other words it can be a credit card, bearing a signature or it could be a door with graffiti written on it. As a budding Crime Scene Investigator, you should know the basics of all types of physical evidence so you don't screw up a scene and lose critical evidentiary value. An example would be the JonBenet Ramsey case where there was a handwritten extortion letter found at the scene. The botched original investigation overlooked many critical details relating to the document. The savvy investigator should have known that there was more to the document than initially appeared to the untrained eye. Once seasoned examiners were called in to assist, it was found that there actually was a previously written extortion note. This note was only partially created, and then the wording was changed when the final note was penned. Also, an unusual pen matching that used to create the document and the actual pad of paper the extortion notes were torn from were both found secreted away within the confines of the house. These missed pieces of evidence could have been critical in the early days of the investigation.

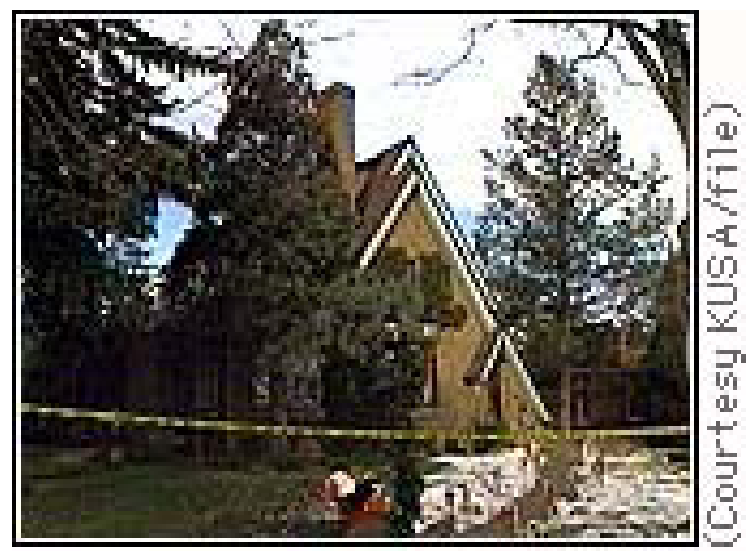

So, now I have to train you to be that savvy investigator. There isn't much time, so let's get to it.

It is critical for the investigator to understand the capability of the forensic laboratory and to conduct an investigation that not only protects a person's rights, but also provides solid steps towards solving the crime.

\section{Document problems}

The most common question when it comes to a document is one of authorship. Did the person sign his or her own name? Other typical document related questions are:

1. The determination of whether erasures or obliterations occurred and whether there is some meaning behind it or whether it was a simple correction of a mistaken entry.
Volume 4 Issue 5 - 2017

\author{
Larry F Stewart \\ Chief Forensic Scientist, Global Forensic Services, USA
}

\begin{abstract}
Correspondence: Larry F Stewart, Chief Forensic Scientist, Global Forensic Services, LLC, USA,
\end{abstract}

Email larry@stewartforensicconsultants.com

Received: April 07, 2017| Published: April 28, 2017

2. Additions to documents, e.g. Has a line been inserted in a written or typed document at a time different from when the document was originally created?
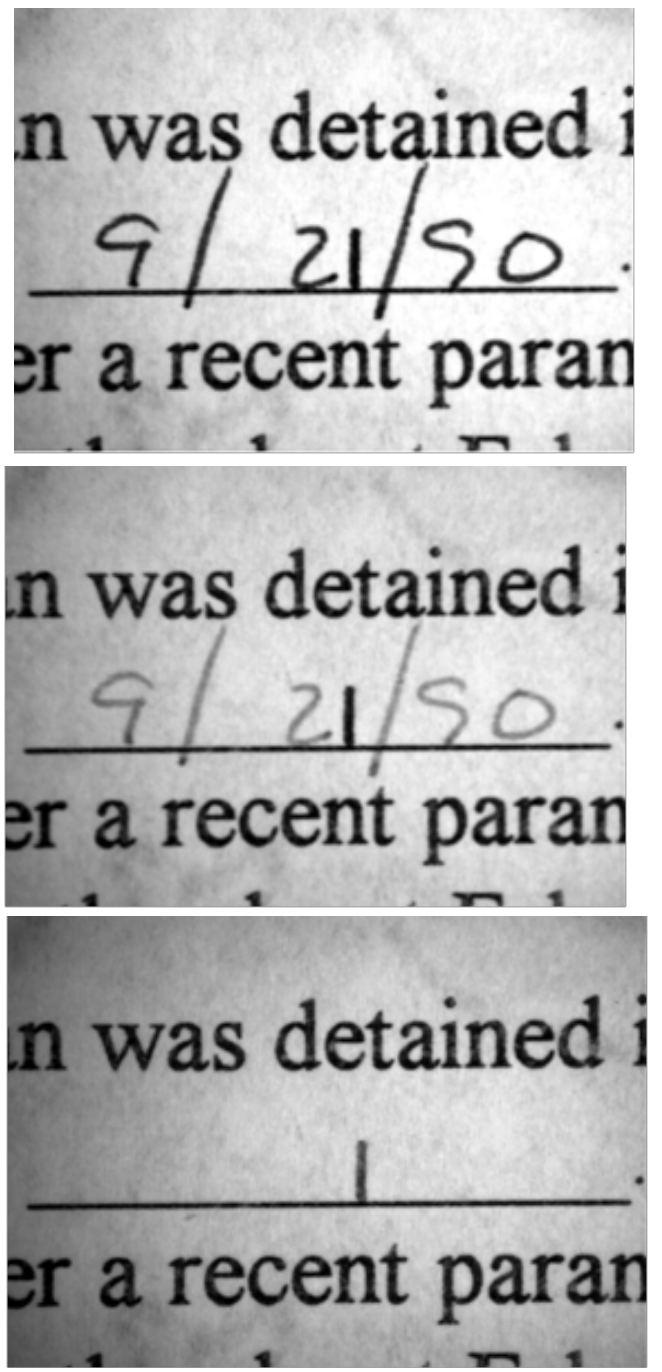
3. Alterations on a document can easily be overlooked by the investigator. They often require microscopic analysis utilizing various light filters usually found in the forensic laboratory for detection. Here is an example of a document photographed 3 different ways, outlining an altered date. It was photographed using dichroic photographic filters, which often can show subtle differences in writing inks.

4. The source of the materials, i.e. pens, paper, typewriter, computer software, etc. Sometimes the uniqueness of the components may be critical to pointing the finger at one suspect.

\section{The age of the document.}

None of these are easy questions and typically can only be addressed in the competent forensic laboratory. However, your steps as the initial investigator are critical to being able to address these questions. One key is to only submit the document related questions to one expert who is capable to make the determination. An unrepairable mistake will be made if you shop around for opinions by taking document related questions to various examiners of differing qualifications. If this exercise yields different conclusions and reports, it is often a source of confusion in the court and one that may lose a case. As the initial investigator on the scene, you must be aware that the value of the document related evidence, as with any physical evidence, diminishes with handling. Keep hands and feet and your own trace evidence away from the scene so as not to contaminate the evidence.

The following suggestions may be of use:

i. Use plastic tongs to handle the document. The worst embarrassment would be for the crime lab to find your fingerprints on the evidence and then you have to explain that contamination of the evidence to the court. Some resources suggest using wooden tongs to pick up documents. I don't, because paper is in part typically made of wood. It could be argued that some transfer of material could occur with the use of wooden tongs, so plastic is best. Also, be concerned over the age and brittleness of the document before picking it up with tongs of any type. Paper documents typically begin their life with around 7 percent of their body weight being composed of water. As the document ages the percentage of water diminishes and the document itself becomes more brittle. Therefore, tongs would not be suggested if a document was purportedly old (10 or more years) or if the document had been exposed to intense heat, e.g. recovered from a fireplace. In these cases other approaches need to be considered such as maintaining the document between two glass plates for transport to the crime lab.

ii. Place the document in an envelope that you previously marked or labeled. Markings should include a unique case and exhibit number, your initials and date. Some investigators insist on marking the actual document. This ahs benefits and drawbacks. If you mark it in an area that ends up having a fingerprint or DNA evidence, you may affect the successful identification. You don't want to mark the envelope after the document has been placed in it because this may add indented writings on the questioned document, also a source of embarrassment for the scene investigator. Likewise, do not place any folds on the document as they may interfere with the examination. A transparent envelope is best, because it will necessitate less opening and resealing of the envelope. Each time the envelope is opened, it must be signed as a point in the chain of custody or ownership of the document.

iii. There has to be an order to the examinations requested of the crime lab. If the document is to be treated for the purposes of developing fingerprints, it may obliterate certain document attributes, e.g. indentations and writing ink analysis. This is because to treat a paper document typically requires chemical spraying or immersion. This action can liquefy and remove ink as well as permanently remove indentations from the paper surface. Therefore, special consideration needs to occur regarding which tests are most important to the case and to protect the document accordingly. Do not spray the document with ninhydrin at the scene to attempt to develop prints, without realizing the potential for loss of other key evidence.

iv. In a separate location as well as on the outer envelope, you should record other pertinent information regarding the location and seizure of the evidence.

In the JonBenet case, indentations should have been checked first before fingerprints as the ninhydrin chemical tests for fingerprints removed the remnants of indentations in the paper. All of the writing pens and pads of paper within the house should have been inventoried and submitted for analysis. Then, it would have been found that a pen typically reserved for artists and artistic drawings, was used to pen both of the extortion notes, This ink composition matched that found in a pen located in the back of a kitchen drawer. The pad of paper that was used was found elsewhere in the house.

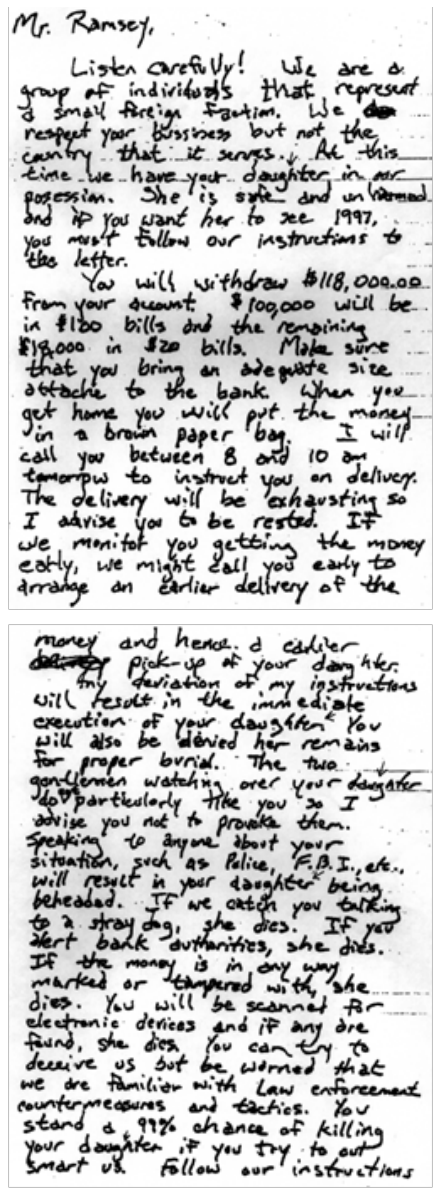




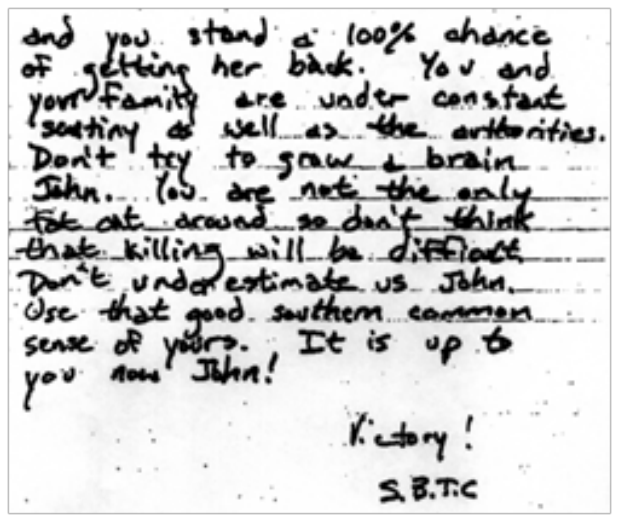

Finally, document related scenes, like all crime scenes must be properly photographed and diagramed by the crime scene investigator. Where was the document found in relation to the various boxes within the scene. By that I mean, start with the outside perimeter of the scene, outlining the area and photographic the layout. This could be the outside of a house and surrounding property, for instance. Next, move to the first inner box, e.g. the rooms within the house that are deemed important by the investigator. Each of these boxes should be drawn and photographed. Then finally, move to the innermost boxes, i.e. the area where the document was found and sketch and photograph. These become your area boxes and can be used to later accurately show where every piece of evidence was found. Through an understanding of crime scene investigations as well as the potential of various types of evidence, the investigator can provide the best opportunity for the forensic laboratory to reach answers.

\section{Acknowledgments}

None.

\section{Conflicts of interest}

The author declares there are no conflicts of interest. 